\title{
CIENCIAMATRIA
}

Revista Interdisciplinaria de Humanidades, Educación, Ciencia y Tecnología

Año VIII. Vol. VIII. N¹4. Enero - Junio. 2022

Hecho el depósito de ley: pp201602FA4721

ISSN-L: 2542-3029; ISSN: 2610-802X

Universidad Nacional Experimental Francisco de Miranda (UNEFM). Santa Ana de Coro. Venezuela

Kenia Lisbeth Alvarado-Cuenca; Ingrid Joselyne Díaz-Basurto; Juan Carlos Arandia-Zambrano

DOI $10.35381 / \mathrm{cm} \cdot \mathrm{v} 8 \mathrm{i} 14.640$

\section{Derecho de adopción homoparental en la legislación ecuatoriana}

\section{Right to homoparental adoption in Ecuadorian legislation}

\author{
Kenia Lisbeth Alvarado-Cuenca \\ dq.keniala36@uniandes.edu.ec \\ Universidad Regional Autónoma de los Andes, Quevedo \\ Ecuador \\ https://orcid.org/0000-0003-1953-3846 \\ Ingrid Joselyne Díaz-Basurto \\ uq.ingriddiaz@uniandes.edu.ec \\ Universidad Regional Autónoma de los Andes, Quevedo \\ Ecuador \\ https://orcid.org/0000-0003-2934-4010 \\ Juan Carlos Arandia-Zambrano \\ uq.juanarandia@uniandes.edu.ec \\ Universidad Regional Autónoma de los Andes, Quevedo \\ Ecuador \\ https://orcid.org/0000-0003-1152-104X
}

Recibido: 15 de septiembre 2021

Revisado: 10 de noviembre 2021

Aprobado: 15 de diciembre 2021

Publicado: 01 de enero 2022 


\section{CIENCIAMATRIA \\ Revista Interdisciplinaria de Humanidades, Educación, Ciencia y Tecnología \\ Año VIII. Vol. VIII. №14. Enero - Junio. 2022 \\ Hecho el depósito de ley: pp201602FA4721 \\ ISSN-L: 2542-3029; ISSN: 2610-802X \\ Universidad Nacional Experimental Francisco de Miranda (UNEFM). Santa Ana de Coro. Venezuela}

Kenia Lisbeth Alvarado-Cuenca; Ingrid Joselyne Díaz-Basurto; Juan Carlos Arandia-Zambrano

\section{Estimado Editor (a):}

En la actualidad, en el marco jurídico ecuatoriano, el derecho de adopción de menores es de carácter limitado y únicamente accesible a parejas de orientación heterosexual, todo ello justificado en base a una sociedad tradicional y consuetudinaria, en la cual no se acepta la idea de formalización de familias mediante la adopción con figuras parentales atípicas en relación a su orientación sexual.

Dentro el marco jurídico ecuatoriano vigente, se desarrolla la prohibición del derecho de adopción de menores a parejas homosexuales, esta afirmación se evidencia claramente en el inciso segundo del artículo 68 de la carta fundamental ecuatoriana en el cual se establece que la adopción únicamente es accesible para parejas de distinto sexo. Esta prohibición vulnera completamente el reconocimiento de los derechos de las personas de orientación sexual diferente a la heterosexual, reconocidos también por la Constitución del Ecuador, hecho que denota una clara vulneración y discriminación hacia los derechos de parejas homosexuales.

Dentro de este contexto, cabe destacar que, la carta fundamental ecuatoriana del año 2008, desarrolla garantías al goce efectivo de todos los derechos para todas las personas sin tener en consideración la orientación sexual, pero siguen existiendo prohibiciones para familias homoparentales en relación al derecho de adopción en comparación con familias heterosexuales, generando claramente vulneraciones al derecho de igualdad de las personas.

En tal sentido, la adopción para parejas homoparentales, ha sido una problemática objeto de estudio, desde hace décadas atrás, puesto que el reconocimiento de parejas homosexuales en las diferentes legislaciones de países del mundo ha traído consigo vulneraciones y discriminaciones en relación al derecho de conformación de una figura familiar, ya que no cabe la adopción en parejas homosexuales, ni de orientación diferente a la heterosexual, aun cuando la carta magna ecuatoriana reconoce a la familia en sus diversas formas. 


\section{CIENCIAMATRIA \\ Revista Interdisciplinaria de Humanidades, Educación, Ciencia y Tecnología \\ Año VIII. Vol. VIII. №14. Enero - Junio. 2022 \\ Hecho el depósito de ley: pp201602FA4721 \\ ISSN-L: 2542-3029; ISSN: 2610-802X \\ Universidad Nacional Experimental Francisco de Miranda (UNEFM). Santa Ana de Coro. Venezuela}

Kenia Lisbeth Alvarado-Cuenca; Ingrid Joselyne Díaz-Basurto; Juan Carlos Arandia-Zambrano

Por otro lado, la figura de homoparentalidad, históricamente ha estado atribuida a varias transformaciones dentro de la cultura oriental: la primera transformación, se manifiesta desde el punto de vista de que dentro del siglo XXI, la nueva generación de personas está siendo educada bajo nuevos valores en base a las formas diferentes de familia que han ido surgiendo con el pasar del tiempo y segundo lugar sobre el hecho que la medicina desde hace más de una década y media, no considera las preferencias sexuales diferentes como enfermedades o como una patología por parte de las ciencias psicológicas.(Chaparro y Guzmán, 2017)

Sin embargo, las autoridades de diferentes países incluida el Estado ecuatoriano han dado paso al reconocimiento del matrimonio igualitario, si esta figura ha sido aceptada a ojos de la ley, este reconocimiento debe ir de la mano del acceso a todos los derechos que una pareja posee, incluido el derecho de adopción de menores. Una de las grandes problemáticas que ha existido a lo largo de los tiempos se ha relacionado directamente con la orientación sexual, puesto que la sociedad a lo largo de la historia ha establecido roles específicos que deben ser cumplidos tanto por hombres como por mujeres. La sociedad posee una clara diferenciación de estereotipos sexuales, hecho por el cual se exige en la sociedad a cada persona que respete el rol sexual que su género establece. (Bolaños y Charry, 2018)

La importancia de la figura de la familia radica en dos aspectos fundamentales en relación a la existencia del ser humano: en primer lugar, la familia brinda protección para el desarrollo de la persona en base a reglas y preceptos aptos para actuar en sociedad. En segundo lugar, la figura de familia brinda la posibilidad de generar comunicación con otros seres humanos, hecho que permite adaptarse a una sociedad determinada que está conformada por obligaciones, derechos y costumbres. (Moran Chumapi, 2017)

Dentro de la sociedad, existen diferentes argumentos en contra de que una pareja homosexual acceda a la adopción de un menor, uno de los argumentos más presente alrededor del mundo es la religión, ya que esta mantiene la ideología de que dios creo al 


\section{CIENCIAMATRIA \\ Revista Interdisciplinaria de Humanidades, Educación, Ciencia y Tecnología \\ Año VIII. Vol. VIII. №14. Enero - Junio. 2022 \\ Hecho el depósito de ley: pp201602FA4721 \\ ISSN-L: 2542-3029; ISSN: 2610-802X \\ Universidad Nacional Experimental Francisco de Miranda (UNEFM). Santa Ana de Coro. Venezuela}

Kenia Lisbeth Alvarado-Cuenca; Ingrid Joselyne Díaz-Basurto; Juan Carlos Arandia-Zambrano

hombre y a la mujer y le impuso un deber divino de procrear y todo lo que sea distinto a eso es inaceptable, sin embargo dentro de cualquier marco jurídico estos fundamentos no tienen solidez, puesto que la ley regula sobre las personas indistintamente de sus inclinaciones sexuales, religiosas o de cualquier índole sin discriminación alguna. (López, 2006)

En concordancia con lo expresado con anterioridad, es importante tomar en cuenta que los modelos de familia desarrollados a lo largo de los años incluyen hijo con madres o padres solteros, viudos, divorciado o están expuestos por diversas circunstancias a la convivencia con abuelos u otros familiares, hecho que no contribuye en mayor o menor medida a su desarrollo como persona o a su inclinación sexual.

Dentro de la legislación ecuatoriana, el acceso al derecho de adopción representa un trámite complicado y de larga duración para quienes tengan la predisposición de adoptar a un menor, situación que se considera como algo normal salvo por una de la características principales descritas en la ley, la cual es el acceso de este derecho únicamente para pareja heterosexuales, el cual evidencia una clara vulneración al derecho de igualdad entre las personas heterosexuales y los homosexuales, contradiciendo lo principios de igualdad que proclama la carta magna ecuatoriana.

Con la finalidad de justificar lo actuado con anterioridad, cabe mencionar lo dispuesto por el artículo 68 de la carta fundamental ecuatoriana, en la cual se establece que la adopción únicamente podrá ser ejecutada por parejas de distinto sexo, evidenciando de esta manera su clara vulneración hacia derechos de igualdad formal y no discriminación. La figura de familia como ya hemos mencionado con anterioridad, desarrolla múltiples formas de existencia, pero todas ellas van apegadas a una estructura muy establecida en sus roles, así pues, en toda familia existe la figura del padre, de la madre y de los hijos, sin cabida a la existencia de dos figuras iguales en los casos de los progenitores, por ello una familia conformada por progenitores de un mismo sexo es inaceptable a ojos de una sociedad que mira la homosexualidad como una enfermedad. 


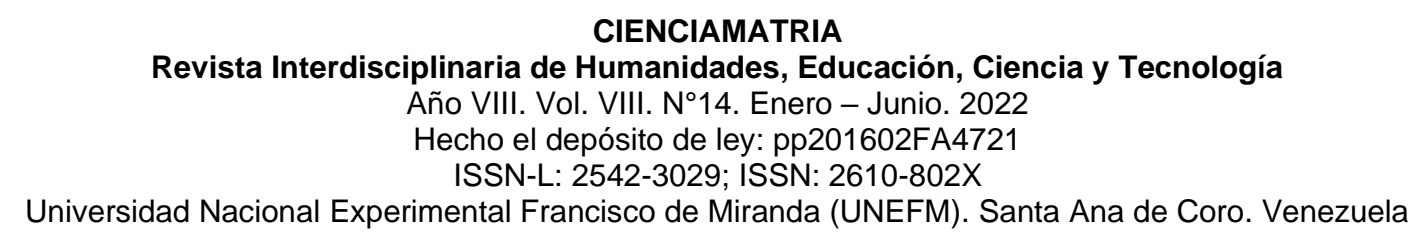

Kenia Lisbeth Alvarado-Cuenca; Ingrid Joselyne Díaz-Basurto; Juan Carlos Arandia-Zambrano

Por lo tanto, la homosexualidad es un tema realmente controvertido, que ha existido, desde el origen de los tiempos, pues a ojos de la sociedad, de la religión y de la costumbre, se espera que toda persona actué en base a su naturaleza humana, como hombre o como mujer, mas no a su orientación sexual, pues si una persona va en contra de su naturaleza, y viola el rol natural que se le ha otorgado, este es considerado como una persona equivocada o antinatural.

En base a estos criterios socioculturales en torno a la familia y a las personas de orientación sexual diferentes a la heterosexual, cabe destacar que en el ámbito del derecho toda persona está amparada bajo el derecho de igualdad ante la ley, sin tener en consideración su orientación o preferencias sexuales, pues el derecho regula comportamientos entre personas indistintamente de su género o preferencias, sin embargo la cruda realidad de la sociedad actual es la existencia de una clara desigualdad y discriminación hacia las personas con orientaciones sexuales diferentes a la heterosexual, hecho que ha generado a lo largo de la historia que las llamadas minorías, en referencia a grupos de orientaciones sexuales diferentes a la tradicional hayan mantenido una lucha exhaustiva para hacer valer sus derechos, para así no ser atentados por el simple hecho de tener una orientación sexual diferente a la ortodoxa.

En definitiva, la legislación ecuatoriana vulnera completamente los derechos de igualdad y no discriminación de parejas homoparentales al existir la prohibición de adopción por parte de estas, así como se genera una vulneración colateral al principio de interés superior del niño, al negarle una posibilidad muy probable de conformar una familia que les permita salir adelante, por ello, es necesario una reforma tanto constitucional como legal para abolir estas vulneraciones, y dar un paso adelante en la lucha por la igualdad de los derechos humanos.

\section{FINANCIAMIENTO}

No monetario. 
CIENCIAMATRIA

Revista Interdisciplinaria de Humanidades, Educación, Ciencia y Tecnología

Año VIII. Vol. VIII. N¹4. Enero - Junio. 2022

Hecho el depósito de ley: pp201602FA4721

ISSN-L: 2542-3029; ISSN: 2610-802X

Universidad Nacional Experimental Francisco de Miranda (UNEFM). Santa Ana de Coro. Venezuela

Kenia Lisbeth Alvarado-Cuenca; Ingrid Joselyne Díaz-Basurto; Juan Carlos Arandia-Zambrano

\section{AGRADECIMIENTO}

A la Universidad Regional Autónoma de los Andes, Quevedo, por motivar el desarrollo de la Investigación.

\section{REFERENCIAS CONSULTADAS}

Asamblea Nacional Constituyente de la República del Ecuador, (2008). Constitución de la República del Ecuador. [Constitution of the Republic of Ecuador]. Montecristi. Registro Oficial 449 de 20-oct-2008. Recuperado de https://n9.cl/sia

Bolaños Enríquez, T., y Charry Morales, A. (2018). Prejuicios y homosexualidad, el largo camino hacia la adopción homoparental. Especial atención al caso colombiano. [Prejudice and homosexuality, the long road to homoparental adoption. Special attention to the Colombian case]. Estudios constitucionales, 16(1), 395-424. https://dx.doi.org/10.4067/S0718-52002018000100395

Chaparro Piedrahíta, L. J., y Guzmán Muñoz, Y. M. (2017). Adopción homoparental: Estudio de derecho comparado a partir de las perspectivas de los países latinoamericanos que la han aprobado. [Homoparental adoption: Comparative law study based on the perspectives of the Latin American countries that have approved it] Revista CES Derecho, (8), 2, 267-297. Recuperado de: https://n9.cl/7zblu

López Sánchez, F. (2006). La adopción por parte de personas homosexuales. [Adoption by homosexual people]. Información Psicológica, 48, 8-20. Recuperado de: https://n9.cl/gtd0m

Moran Chumapi, A. (2017). La adopción en parejas del mismo sexo el derecho a la familia e igualdad. [Adoption in same-sex couples the right to family and equality]. Proyecto de investigación. Universidad Regional Autónoma de los Andes. Ambato. Ecuador. Recuperado de: https://n9.cl/97dh6

(C2022 por los autores. Este artículo es de acceso abierto y distribuido según los términos y condiciones de la licencia Creative Commons Atribución-NoComercial-Compartirlgual 4.0 Internacional (CC BY-NC-SA 4.0)

(https://creativecommons.org/licenses/by-nc-sa/4.0/). 